\title{
A GUIDE TO ENTROPY AND THE SECOND LAW OF THERMODYNAMICS
}

\author{
Elliott H. Lieb* \\ Departments of Mathematics and Physics, Princeton University \\ Jadwin Hall, P.O. Box 708, Princeton, NJ 08544, USA \\ Jakob Yngvason ** \\ Institut für Theoretische Physik, Universität Wien, \\ Boltzmanngasse 5, A 1090 Vienna, Austria
}

This article is intended for readers who, like us, were told that the second law of thermodynamics is one of the major achievements of the nineteenth century, that it is a logical, perfect and unbreakable law - but who were unsatisfied with the 'derivations' of the entropy principle as found in textbooks and in popular writings.

A glance at the books will inform the reader that the law has 'various formulations' (which is a bit odd, as if to say the ten commandments have various formulations) but they all lead to the existence of an entropy function whose reason for existence is to tell us which processes can occur and which cannot. We shall abuse language (or reformulate it) by referring to the existence of entropy as the second law. This, at least, is unambiguous. The entropy we are talking about is that defined by thermodynamics (and not some analytic quantity, usually involving expressions such as $-p \ln p$, that appears in information theory, probability theory and statistical mechanical models).

There are three laws of thermodynamics (plus one more, due to Nernst, which is mainly used in low temperature physics and is not immutable like the others). In brief, these are:

The Zeroth Law, which expresses the transitivity of equilibrium, and which is often said to imply the existence of temperature as a parametrization of equilibrium states. We use it below but formulate it without mentioning temperature. In fact, temperature makes no appearance here until almost the very end.

The First Law, which is conservation of energy. It is a concept from mechanics and provides the connection between mechanics (and things like falling weights) and thermodynamics. We discuss this later on when we introduce simple systems; the crucial

\footnotetext{
* Work partially supported by U.S. National Science Foundation grant PHY95-13072A01.

** Work partially supported by the Adalsteinn Kristjansson Foundation, University of Iceland.

(c) 1997 by the authors. Reproduction of this article, by any means, is permitted for non-commercial purposes.
} 
usage of this law is that it allows energy to be used as one of the parameters describing the states of a simple system.

The Second Law. Three popular formulations of this law are:

Clausius: No process is possible, the sole result of which is that heat is transferred from a body to a hotter one.

Kelvin (and Planck): No process is possible, the sole result of which is that a body is cooled and work is done.

Carathéodory: In any neighborhood of any state there are states that cannot be reached from it by an adiabatic process.

All three are supposed to lead to the entropy principle (defined below). These steps can be found in many books and will not be trodden again here. Let us note in passing, however, that the first two use concepts such as hot, cold, heat, cool that are intuitive but have to be made precise before the statements are truly meaningful. No one has seen 'heat', for example. The last (which uses the term "adiabatic process", to be defined below) presupposes some kind of parametrization of states by points in $\mathbf{R}^{n}$, and the usual derivation of entropy from it assumes some sort of differentiability; such assumptions are beside the point as far as understanding the meaning of entropy goes.

Why, one might ask, should a mathematician be interested in this matter, which, historically, had something to do with attempts to understand and improve the efficiency of steam engines? The answer, as we perceive it, is that the law is really an interesting mathematical theorem about orderings on sets, with profound physical implications. The axioms that constitute this ordering are somewhat peculiar from the mathematical point of view and might not arise in the ordinary ruminations of abstract thought. They are special, but important, and they are driven by considerations about the world, which is what makes them so interesting. Maybe an ingenious reader will find an application of this same logical structure to another field of science.

The basic input in our analysis is a certain kind of ordering on a set and denoted by

(pronounced 'precedes'). It is transitive and reflexive as in A1, A2 below, but $X \prec Y$ and $Y \prec X$ does not imply $X=Y$, so it is a 'preorder'. The big question is whether $\prec$ can be encoded in an ordinary, real-valued function on the set, denoted by $S$, such that if $X$ and $Y$ are related by $\prec$, then $S(X) \leq S(Y)$ if and only if $X \prec Y$. The function $S$ is also required to be additive and extensive in a sense that will soon be made precise.

A helpful analogy is the question: When can a vector-field, $V(x)$, on $\mathbf{R}^{3}$ be encoded in an ordinary function, $f(x)$, whose gradient is $V$ ? The well-known answer is that a necessary 
and sufficient condition is that $\operatorname{curl} V=0$. Once $V$ is observed to have this property one thing becomes evident and important: It is necessary to measure the integral of $V$ only along some curves - not all curves - in order to deduce the integral along all curves. The encoding then has enormous predictive power about the nature of future measurements of $V$. In the same way, knowledge of the function $S$ has enormous predictive power in the hands of chemists, engineers and others concerned with the ways of the physical world.

Our concern will be the existence and properties of $S$, starting from certain natural axioms about the relation $\prec$. We present our results without proofs, but full details, and a discussion of related previous work on the foundations of classical thermodynamics, are given in [7]. The literature on this subject is extensive and it is not possible to give even a brief account of it here, except for mentioning that the previous work closest to ours is that of [6], and [2], (see also [4], [5] and [9]). These other approaches are also based on an investigation of the relation $\prec$, but the overlap with our work is only partial. In fact, a major part of our work is the derivation of a certain property (the "comparison hypothesis" below), which is taken as an axiom in the other approaches. It was a remarkable and largely unsung achievement of Giles [6] to realize the full power of this property.

Let us begin the story with some basic concepts.

1. Thermodynamic System: Physically, this consists of certain specified amounts of certain kinds of matter, e.g., a gram of hydrogen in a container with a piston, or a gram of hydrogen and a gram of oxygen in two separate containers, or a gram of hydrogen and two grams of hydrogen in separate containers. The system can be in various states which, physically, are equilibrium states. The space of states of the system is usually denoted by a symbol such as $\Gamma$ and states in $\Gamma$ by $X, Y, Z$, etc.

Physical motivation aside, a state-space, mathematically, is just a set — to begin with; later on we will be interested in embedding state-spaces in some convex subset of some $\mathbf{R}^{n+1}$, i.e., we will introduce coordinates. As we said earlier, however, the entropy principle is quite independent of coordinatization, Carathéodory's principle notwithstanding.

2. Composition and scaling of states: The notion of Cartesian product, $\Gamma_{1} \times \Gamma_{2}$ corresponds simply to the two (or more) systems being side by side on the laboratory table; mathematically it is just another system (called a compound system), and we regard the state space $\Gamma_{1} \times \Gamma_{2}$ as the same as $\Gamma_{2} \times \Gamma_{1}$. Points in $\Gamma_{1} \times \Gamma_{2}$ are denoted by pairs $(X, Y)$, as usual. The subsystems comprising a compound system are physically independent systems, but they are allowed to interact with each other for a period of time and thereby alter each other's state.

The concept of scaling is crucial. It is this concept that makes our thermodynamics inappropriate for microscopic objects like atoms or cosmic objects like stars. For each state-space $\Gamma$ and number $\lambda>0$ there is another state-space, denoted by $\Gamma^{(\lambda)}$ with 
points denoted by $\lambda X$. This space is called a scaled copy of $\Gamma$. Of course we identify $\Gamma^{(1)}=\Gamma$ and $1 X=X$. We also require $\left(\Gamma^{(\lambda)}\right)^{(\mu)}=\Gamma^{(\lambda \mu)}$ and $\mu(\lambda X)=(\mu \lambda) X$. The physical interpretation of $\Gamma^{(\lambda)}$ when $\Gamma$ is the space of one gram of hydrogen, is simply the state-space of $\lambda$ grams of hydrogen. The state $\lambda X$ is the state of $\lambda$ grams of hydrogen with the same 'intensive' properties as $X$, e.g., pressure, while 'extensive' properties like energy, volume etc. are scaled by a factor $\lambda$, (by definition).

For any given $\Gamma$ we can form Cartesian product state spaces of the type $\Gamma^{\left(\lambda_{1}\right)} \times \Gamma^{\left(\lambda_{2}\right)} \times$ $\cdots \times \Gamma^{\left(\lambda_{N}\right)}$. These will be called multiple scaled copies of $\Gamma$.

The notation $\Gamma^{(\lambda)}$ should be regarded as merely a mnemonic at this point, but later on, with the embedding of $\Gamma$ into $\mathbf{R}^{n+1}$, it will literally be $\lambda \Gamma=\{\lambda X: X \in \Gamma\}$ in the usual sense.

3. Adiabatic accessibility: Now we come to the ordering. We say $X \prec Y$ (with $X$ and $Y$ possibly in different state-spaces) if there is an adiabatic process that transforms $X$ into $Y$.

What does this mean? Mathematically, we are just given a list of pairs $X \prec Y$. There is nothing more to be said, except that later on we will assume that this list has certain properties that will lead to interesting theorems about this list, and will lead, in turn, to the existence of an entropy function, $S$ characterizing the list.

The physical interpretation is quite another matter. In text books a process is usually called adiabatic if it takes place in 'thermal isolation', which in turn means that 'no heat is exchanged with the surroundings'. Such statements appear neither sufficiently general nor precise to us and we prefer the following version (which is in the spirit of Planck's formulation of the second law [8]). It has the great virtue (as discovered by Planck) that it avoids having to distinguish between work and heat - or even having to define the concept of heat. We emphasize, however, that the theorems do not require agreement with our physical definition of adiabatic process; other definitions are conceivably possible.

A state $Y$ is adiabatically accessible from a state $X$, in symbols $X \prec Y$, if it is possible to change the state from $X$ to $Y$ by means of an interaction with some device consisting of some auxiliary system and a weight, in such a way that the auxiliary system returns to its initial state at the end of the process whereas the weight may have risen or fallen.

The role of the 'weight' in this definition is merely to provide a particularly simple source (or sink) of mechanical energy. Note that an adiabatic process, physically, does not have to be gentle, or 'static' or anything of the kind. It can be arbitrarily violent! 

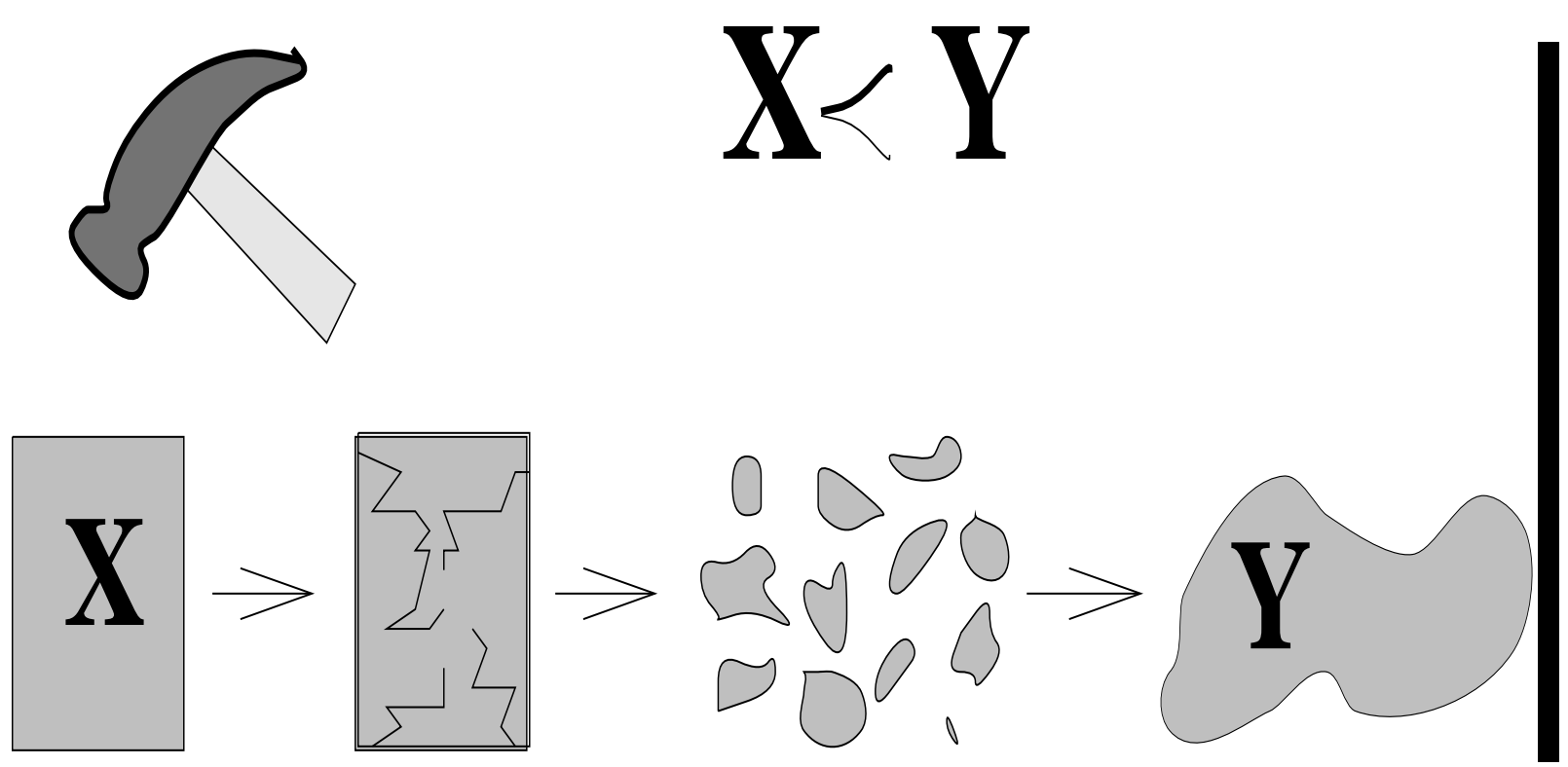

Figure 1. A violent adiabatic process connecting equilibrium states $X$ and $Y$.

An example might be useful here. Take a pound of hydrogen in a container with a piston. The states are describable by two numbers, energy and volume, the latter being determined by the position of the piston. Starting from some state, $X$, we can take our hand off the piston and let the volume increase explosively to a larger one. After things have calmed down, call the new equilibrium state $Y$. Then $X \prec Y$. Question: Is $Y \prec X$ true? Answer: No. To get from $Y$ to $X$ we would have to use some machinery and a weight, with the machinery returning to its initial state, and there is no way this can be done. Using a weight we can, indeed, recompress the gas to its original volume, but we will find that the energy is then larger than its original value.

Let us write

$$
X \prec \prec Y \quad \text { if } \quad X \prec Y \quad \text { but not } Y \prec X \text { (written } Y \nprec X) \text {. }
$$

In this case we say that we can go from $X$ to $Y$ by an irreversible adiabatic process. If $X \prec Y$ and $Y \prec X$ we say that $X$ and $Y$ are adiabatically equivalent and write

$$
X \stackrel{\mathrm{A}}{\sim} Y .
$$

Equivalence classes under $\stackrel{\mathrm{A}}{\sim}$ are called adiabats.

4. Comparability: Given two states $X$ and $Y$ in two (same or different) state-spaces, we say that they are comparable if $X \prec Y$ or $Y \prec X$ (or both). This turns out to be a 
crucial notion. Two states are not always comparable; a necessary condition is that they have the same material composition in terms of the chemical elements. Example: Since water is $\mathrm{H}_{2} \mathrm{O}$ and the atomic weights of hydrogen and oxygen are 1 and 16 respectively, the states in the compound system of 2 gram of hydrogen and 16 grams of oxygen are comparable with states in a system consisting of 18 grams of water (but not with 11 grams of water or 18 grams of oxygen).

Actually, the classification of states into various state-spaces is done mainly for conceptual convenience. The second law deals only with states, and the only thing we really have to know about any two of them is whether or not they are comparable. Given the relation $\prec$ for all possible states of all possible systems, we can ask whether this relation can be encoded in an entropy function according to the following:

Entropy principle: There is a real-valued function on all states of all systems (including compound systems), called entropy and denoted by S such that

a) Monotonicity: When $X$ and $Y$ are comparable states then

$$
X \prec Y \quad \text { if and only if } \quad S(X) \leq S(Y)
$$

b) Additivity and extensivity: If $X$ and $Y$ are states of some (possibly different) systems and if $(X, Y)$ denotes the corresponding state in the compound system, then the entropy is additive for these states, i.e.,

$$
S(X, Y)=S(X)+S(Y)
$$

$S$ is also extensive, i.e., for or each $\lambda>0$ and each state $X$ and its scaled copy $\lambda X \in \Gamma^{(\lambda)}$, (defined in 2. above)

$$
S(\lambda X)=\lambda S(X)
$$

A formulation logically equivalent to a), not using the word 'comparable', is the following pair of statements:

$$
\begin{aligned}
X \stackrel{\mathrm{A}}{\sim} Y & \Longrightarrow S(X)=S(Y) \quad \text { and } \\
X \prec \prec Y & \Longrightarrow S(X)<S(Y) .
\end{aligned}
$$

The last line is especially noteworthy. It says that entropy must increase in an irreversible adiabatic process.

The additivity of entropy in compound systems is often just taken for granted, but it is one of the startling conclusions of thermodynamics. First of all, the content of additivity, 
(2), is considerably more far reaching than one might think from the simplicity of the notation. Consider four states $X, X^{\prime}, Y, Y^{\prime}$ and suppose that $X \prec Y$ and $X^{\prime} \prec Y^{\prime}$. One of our axioms, A3, will be that then $\left(X, X^{\prime}\right) \prec\left(Y, Y^{\prime}\right)$, and (2) contains nothing new or exciting. On the other hand, the compound system can well have an adiabatic process in which $\left(X, X^{\prime}\right) \prec\left(Y, Y^{\prime}\right)$ but $X \nprec Y$. In this case, (2) conveys much information. Indeed, by monotonicity, there will be many cases of this kind because the inequality $S(X)+S\left(X^{\prime}\right) \leq S(Y)+S\left(Y^{\prime}\right)$ certainly does not imply that $S(X) \leq S(Y)$. The fact that the inequality $S(X)+S\left(X^{\prime}\right) \leq S(Y)+S\left(Y^{\prime}\right)$ tells us exactly which adiabatic processes are allowed in the compound system (among comparable states), independent of any detailed knowledge of the manner in which the two systems interact, is astonishing and is at the heart of thermodynamics. The second reason that (2) is startling is this: From (1) alone, restricted to one system, the function $S$ can be replaced by $29 S$ and still do its job, i.e., satisfy (1). However, (2) says that it is possible to calibrate the entropies of all systems (i.e., simultaneously adjust all the undetermined multiplicative constants) so that the entropy $S_{1,2}$ for a compound $\Gamma_{1} \times \Gamma_{2}$ is $S_{1,2}(X, Y)=S_{1}(X)+S_{2}(Y)$, even though systems 1 and 2 are totally unrelated!

We are now ready to ask some basic questions:

Q1: Which properties of the relation $\prec$ ensure existence and (essential) uniqueness of $S$ ?

Q2: Can these properties be derived from simple physical premises?

Q3: Which convexity and smoothness properties of $S$ follow from the premises?

Q4: Can temperature (and hence an ordering of states by "hotness" and "coldness") be defined from $S$ and what are its properties?

The answer to question Q1 can be given in the form of six axioms that are reasonable, simple, 'obvious' and unexceptionable. An additional, crucial assumption is also needed, but we call it a 'hypothesis' instead of an axiom because we show later how it can be derived from some other axioms, thereby answering question Q2.

A1. Reflexivity. $X \stackrel{\wedge}{\sim} X$.

A2. Transitivity. If $X \prec Y$ and $Y \prec Z$, then $X \prec Z$.

A3. Consistency. If $X \prec X^{\prime}$ and $Y \prec Y^{\prime}$, then $(X, Y) \prec\left(X^{\prime}, Y^{\prime}\right)$.

A4. Scaling Invariance. If $\lambda>0$ and $X \prec Y$, then $\lambda X \prec \lambda Y$.

A5. Splitting and Recombination. $X \stackrel{\mathrm{A}}{\sim}((1-\lambda) X, \lambda X)$ for all $0<\lambda<1$. Note that the state-space are not the same on both sides. If $X \in \Gamma$, then the state space on the right side is $\Gamma^{(1-\lambda)} \times \Gamma^{(\lambda)}$. 
A6. Stability. If $\left(X, \varepsilon Z_{0}\right) \prec\left(Y, \varepsilon Z_{1}\right)$ for some $Z_{0}, Z_{1}$ and a sequence of $\varepsilon$ 's tending to zero, then $X \prec Y$. This axiom is a substitute for continuity, which we cannot assume because there is no topology yet. It says that 'a grain of dust cannot influence the set of adiabatic processes'.

An important lemma is that (A1)-(A6) imply the cancellation law, which is used in many proofs. It says that for any three states $X, Y, Z$

$$
(X, Z) \prec(Y, Z) \Longrightarrow X \prec Y
$$

The next concept plays a key role in our treatment.

CH. Definition: We say that the Comparison Hypothesis, $(\mathrm{CH})$, holds for a state-space $\Gamma$ if all pairs of states in $\Gamma$ are comparable.

Note that A3, A4 and A5 automatically extend comparability from a space $\Gamma$ to certain other cases, e.g., $X \prec((1-\lambda) Y, \lambda Z)$ for all $0 \leq \lambda \leq 1$ if $X \prec Y$ and $X \prec Z$. On the other hand, comparability on $\Gamma$ alone does not allow us to conclude that $X$ is comparable to $((1-\lambda) Y, \lambda Z)$ if $X \prec Y$ but $Z \prec X$. For this, one needs $\mathrm{CH}$ on the product space $\Gamma^{(1-\lambda)} \times \Gamma^{(\lambda)}$, which is not implied by $\mathrm{CH}$ on $\Gamma$.

The significance of $\mathrm{A} 1-\mathrm{A} 6$ and $\mathrm{CH}$ is borne out by the following theorem:

THEOREM 1 (Equivalence of entropy and A1-A6, given CH). The following are equivalent for a state-space $\Gamma$ :

(i) The relation $\prec$ between states in (possibly different) multiple scaled copies of $\Gamma$ e.g., $\Gamma^{\left(\lambda_{1}\right)} \times \Gamma^{\left(\lambda_{2}\right)} \times \cdots \times \Gamma^{\left(\lambda_{N}\right)}$, is characterized by an entropy function, $S$, on $\Gamma$ in the sense that

$$
\left(\lambda_{1} X_{1}, \lambda_{2} X_{2}, \ldots\right) \prec\left(\lambda_{1}^{\prime} X_{1}^{\prime}, \lambda_{2}^{\prime} X_{2}^{\prime}, \ldots\right)
$$

is equivalent to the condition that

$$
\sum_{i} \lambda_{i} S\left(X_{i}\right) \leq \sum_{j} \lambda_{j}^{\prime} S\left(X_{j}^{\prime}\right)
$$

whenever

$$
\sum_{i} \lambda_{i}=\sum_{j} \lambda_{j}^{\prime}
$$

(ii) The relation $\prec$ satisfies conditions (A1)-(A6), and (CH) holds for every multiple scaled copy of $\Gamma$.

This entropy function on $\Gamma$ is unique up to affine equivalence, i.e., $S(X) \rightarrow a S(X)+B$, with $a>0$. 
That (i) $\Longrightarrow$ (ii) is obvious. The proof of (ii) $\Longrightarrow$ (i) is carried out by an explicit construction of the entropy function on $\Gamma$-reminiscent of an old definition of heat by Laplace and Lavoisier in terms of the amount of ice that a body can melt.

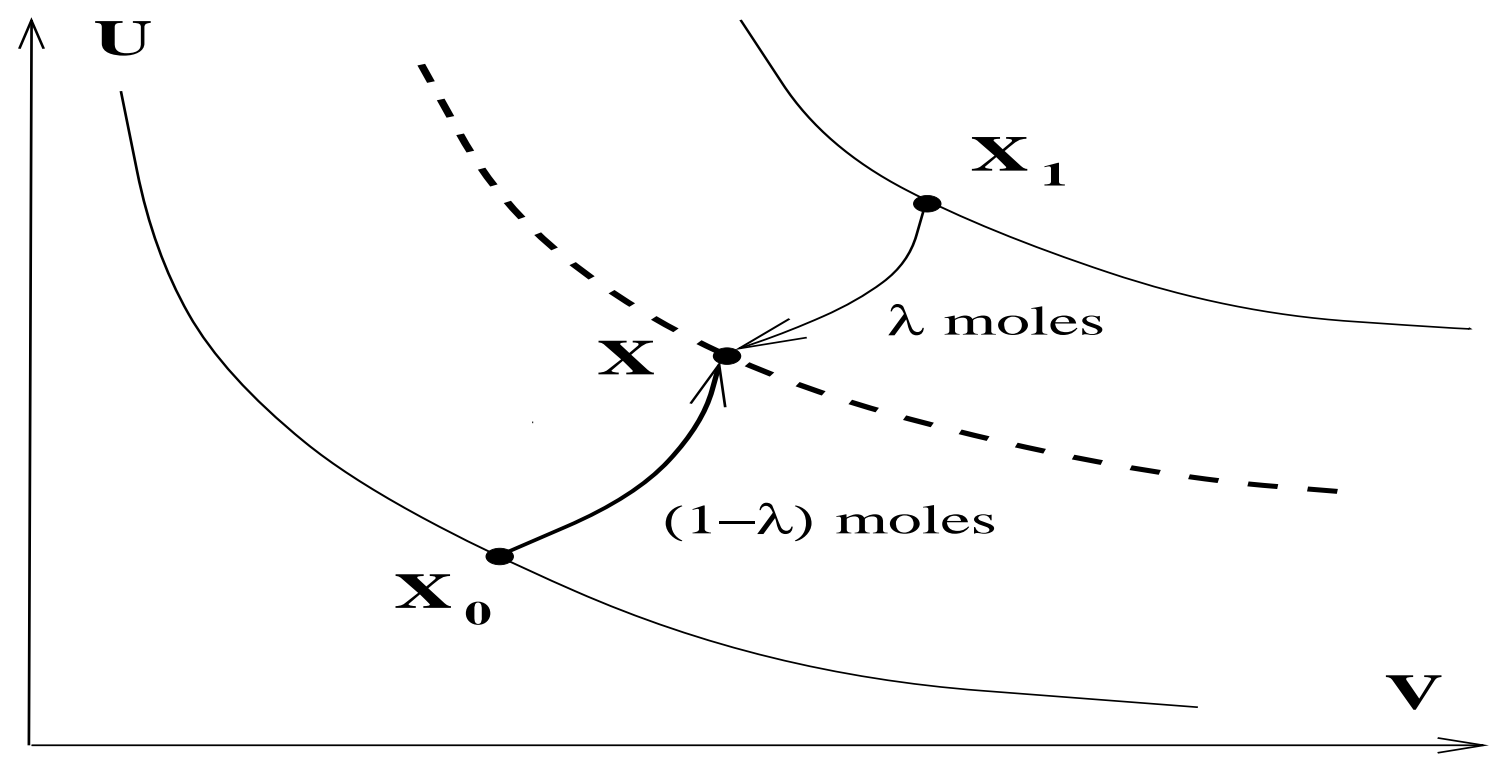

2. The entropy of $X$ is determined by the largest amount of $X_{1}$ that can be transformed adiabatically into $X$, with the help of $X_{0}$.

BASIC CONSTRUCTION of $S$ : Pick two reference points $X_{0}$ and $X_{1}$ in $\Gamma$ with $X_{0} \prec \prec X_{1}$. (If such points do not exist then $S$ is the constant function.) Then define for $X \in \Gamma$

$$
S(X):=\sup \left\{\lambda:\left((1-\lambda) X_{0}, \lambda X_{1}\right) \prec X\right\}
$$

Remarks: As in axiom A5, two state-spaces are involved in (9). By axiom A5, $X \stackrel{\text { A }}{\sim}$ $((1-\lambda) X, \lambda X)$, and hence, by $\mathrm{CH}$ in the space $\Gamma^{(1-\lambda)} \times \Gamma^{(\lambda)}, X$ is comparable to $((1-$ $\left.\lambda) X_{0}, \lambda X_{1}\right)$. In (9) we allow $\lambda \leq 0$ and $\lambda \geq 1$ by using the convention that $(X,-Y) \prec Z$ means that $X \prec(Y, Z)$ and $(X, 0 Y)=X$. For (9) we only need to know that $\mathrm{CH}$ holds in two-fold scaled products of $\Gamma$ with itself. $\mathrm{CH}$ will then automatically be true for all products. In (9) the reference points $X_{0}, X_{1}$ are fixed and the supremum is over $\lambda$. One can ask how $S$ changes if we change the two points $X_{0}, X_{1}$. The answer is that the change is affine, i.e., $S(X) \rightarrow a S(X)+B$, with $a>0$.

Theorem 1 extends to products of multiple scaled copies of different systems, i.e. to general compound systems. This extension is an immediate consequence of the following theorem, which is proved by applying Theorem 1 to the product of the system under consideration with some standard reference system. 
THEOREM 2 (Consistent entropy scales). Assume that $C H$ holds for all compound systems. For each system $\Gamma$ let $S_{\Gamma}$ be some definite entropy function on $\Gamma$ in the sense of Theorem 1. Then there are constants $a_{\Gamma}$ and $B(\Gamma)$ such that the function $S$, defined for all states of all systems by

$$
S(X)=a_{\Gamma} S_{\Gamma}(X)+B(\Gamma)
$$

for $X \in \Gamma$, satisfies additivity (2), extensivity (3), and monotonicity (1) in the sense that whenever $X$ and $Y$ are in the same state space then

$$
X \prec Y \quad \text { if and only if } \quad S(X) \leq S(Y) .
$$

Theorem 2 is what we need, except for the question of mixing and chemical reactions, which is treated at the end and which can be put aside at a first reading. In other words, as long as we do not consider adiabatic processes in which systems are converted into each other (e.g., a compound system consisting of vessel of hydrogen and a vessel of oxygen is converted into a vessel of water), the entropy principle has been verified. If that is so, what remains to be done, the reader may justifiably ask? The answer is twofold: First, Theorem 2 requires that $\mathrm{CH}$ holds for all systems, and we are not content to take this as an axiom. Second, important notions of thermodynamics such as 'thermal equilibrium' (which will eventually lead to a precise definition of 'temperature' ) have not appeared so far. We shall see that these two points (i.e., thermal equilibrium and $\mathrm{CH}$ ) are not unrelated.

As for $\mathrm{CH}$, other authors, [6], [2], [4] and [9] essentially postulate that it holds for all systems by making it axiomatic that comparable states fall into equivalence classes. (This means that the conditions $X \prec Z$ and $Y \prec Z$ always imply that $X$ and $Y$ are comparable: likewise, they must be comparable if $Z \prec X$ and $Z \prec Y$ ). By identifying a 'state-space' with an equivalence class, the comparison hypothesis then holds in these other approaches by assumption for all state-spaces. We, in contrast, would like to derive $\mathrm{CH}$ from something that we consider more basic. Two ingredients will be needed: The analysis of certain special, but commonplace systems called 'simple systems' and some assumptions about thermal contact (the 'Zeroth law') that will act as a kind of glue holding the parts of a compound systems in harmony with each other.

A Simple System is one whose state-space can be identified with some open convex subset of some $\mathbf{R}^{n+1}$ with a distinguished coordinate denoted by $U$, called the energy, and additional coordinates $V \in \mathbf{R}^{n}$, called work coordinates. The energy coordinate is the way in which thermodynamics makes contact with mechanics, where the concept of energy arises and is precisely defined. The fact that the amount of energy in a state is independent of the manner in which the state was arrived at is, in reality, the first law of thermodynamics. A typical (and often the only) work coordinate is the volume of a fluid or gas (controlled by a piston); other examples are deformation coordinates of a solid or magnetization of a paramagnetic substance. 
Our goal is to show, with the addition of a few more axioms, that $\mathrm{CH}$ holds for simple systems and their scaled products. In the process, we will introduce more structure, which will capture the intuitive notions of thermodynamics; thermal equilibrium is one.

First, there is an axiom about convexity:

A7. Convex combination. If $X$ and $Y$ are states of a simple system and $t \in[0,1]$ then

$$
(t X,(1-t) Y) \prec t X+(1-t) Y
$$

in the sense of ordinary convex addition of points in $\mathbf{R}^{n+1}$. A straightforward consequence of this axiom (and A5) is that the forward sectors

$$
A_{X}:=\{Y \in \Gamma: X \prec Y\}
$$

of states $X$ in a simple system $\Gamma$ are convex sets.

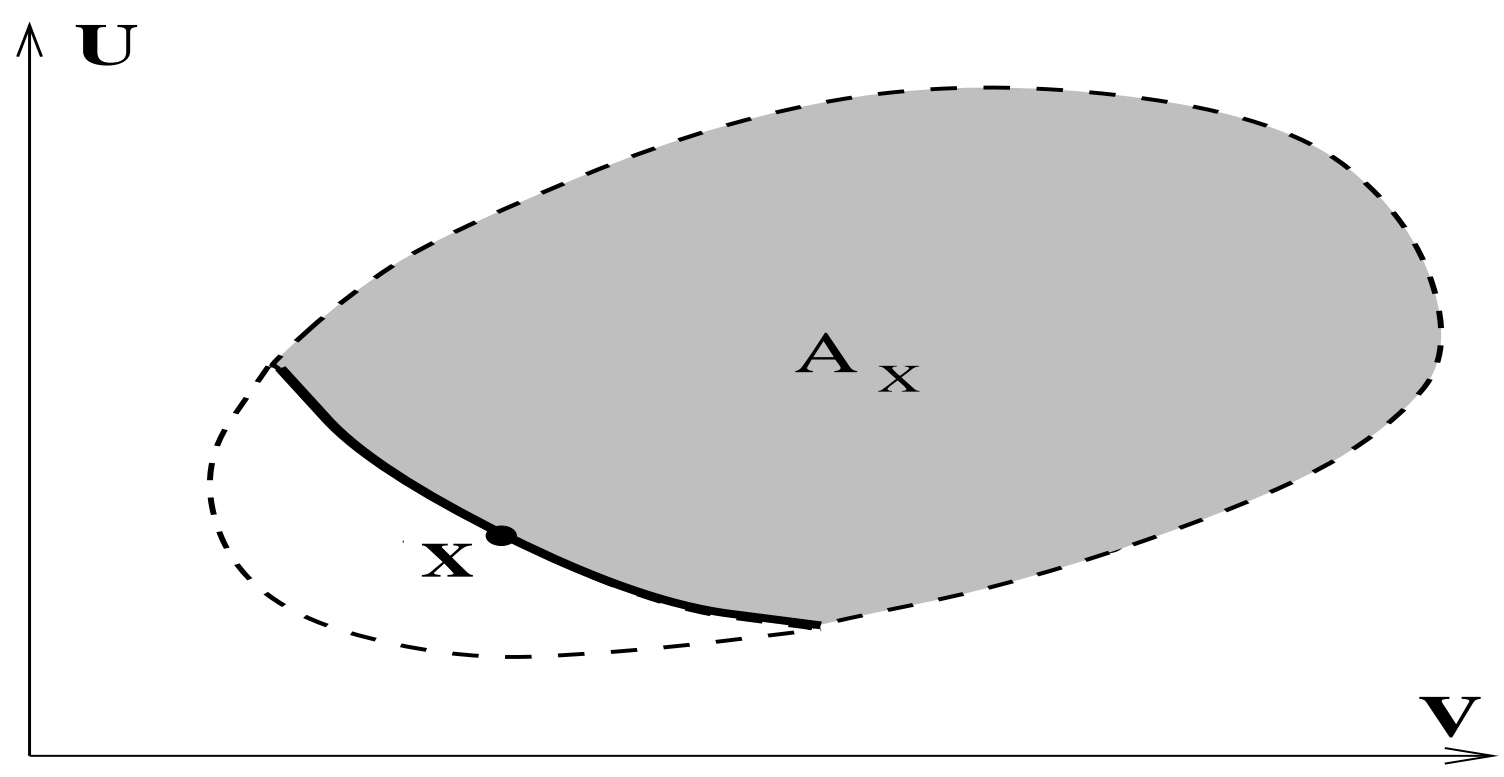

3. The coordinates $U$ and $V$ of a simple system. The state space (bounded by dashed line) and the forward sector $A_{X}$ (shaded) of a state $X$ are convex, by axiom A7. The boundary of $A_{X}$ (full line) is an adiabat. 
Another consequence is a connection between the existence of irreversible processes and Carathéodory's principle ([3], [1]) mentioned above.

LEMMA 1. Assume (A1)-(AY) for $\Gamma \subset R^{N}$ and consider the following statements:

(a). Existence of irreversible processes: For every $X \in \Gamma$ there is a $Y \in \Gamma$ with $X \prec \prec Y$.

(b). Carathéodory's principle: In every neighborhood of every $X \in \Gamma$ there is a $Z \in \Gamma$ with $X \nprec Z$.

Then $(a) \Longrightarrow(b)$ always. If the forward sectors in $\Gamma$ have interior points, then $(b) \Longrightarrow$ (a).

We need three more axioms for simple systems, which will take us into an analytic detour. The first of these establishes (a) above.

A8. Irreversibility. For each $X \in \Gamma$ there is a point $Y \in \Gamma$ such that $X \prec \prec Y$. (This axiom is implied by A14 below, but is stated here separately because important conclusions can be drawn from it alone.)

A9. Lipschitz tangent planes. For each $X \in \Gamma$ the forward sector $A_{X}=\{Y \in \Gamma$ : $X \prec Y\}$ has a unique support plane at $X$ (i.e., $A_{X}$ has a tangent plane at $X$ ). The tangent plane is assumed to be a locally Lipschitz continuous function of $X$, in the sense explained below.

A10. Connectedness of the boundary. The boundary $\partial A_{X}$ (relative to the open set $\Gamma$ ) of every forward sector $A_{X} \subset \Gamma$ is connected. (This is technical and conceivably can be replaced by something else.)

Axiom A8 plus Lemma 1 asserts that every $X$ lies on the boundary $\partial A_{X}$ of its forward sector. Although axiom A9 asserts that the convex set, $A_{X}$, has a true tangent at $X$ only, it is an easy consequence of axiom A2 that $A_{X}$ has a true tangent everywhere on its boundary. To say that this tangent plane is locally Lipschitz continuous means that if $X=\left(U^{0}, V^{0}\right)$ then this plane is given by

$$
U-U^{0}+\sum_{1}^{n} P_{i}(X)\left(V_{i}-V_{i}^{0}\right)=0
$$

with locally Lipschitz continuous functions $P_{i}$. The function $P_{i}$ is called the generalized pressure conjugate to the work coordinate $V_{i}$. (When $V_{i}$ is the volume, $P_{i}$ is the ordinary pressure.)

Lipschitz continuity and connectedness is a well known guarantee that the coupled differential equations

$$
\frac{\partial U}{\partial V_{j}}(V)=-P_{j}(U(V), V) \text { for } j=1, \ldots, n .
$$


not only have a solution (since we know that the surface $\partial A_{X}$ exists) but this solution must be unique. Thus, if $Y \in \partial A_{X}$ then $X \in \partial A_{Y}$. In short, the surfaces $\partial A_{X}$ foliate the state-space $\Gamma$. What is less obvious, but very important because it instantly gives us the comparison hypothesis for $\Gamma$, is the following.

THEOREM 3 (Forward sectors are nested). If $A_{X}$ and $A_{Y}$ are two forward sectors in the state-space, $\Gamma$, of a simple system then exactly one of the following holds.

(a). $A_{X}=A_{Y}$, i.e., $X \stackrel{\mathrm{A}}{\sim} Y$.

(b). $A_{X} \subset \operatorname{Interior}\left(A_{Y}\right)$, i.e., $Y \prec \prec X$

(c). $A_{Y} \subset \operatorname{Interior}\left(A_{X}\right)$, i.e., $X \prec \prec Y$.
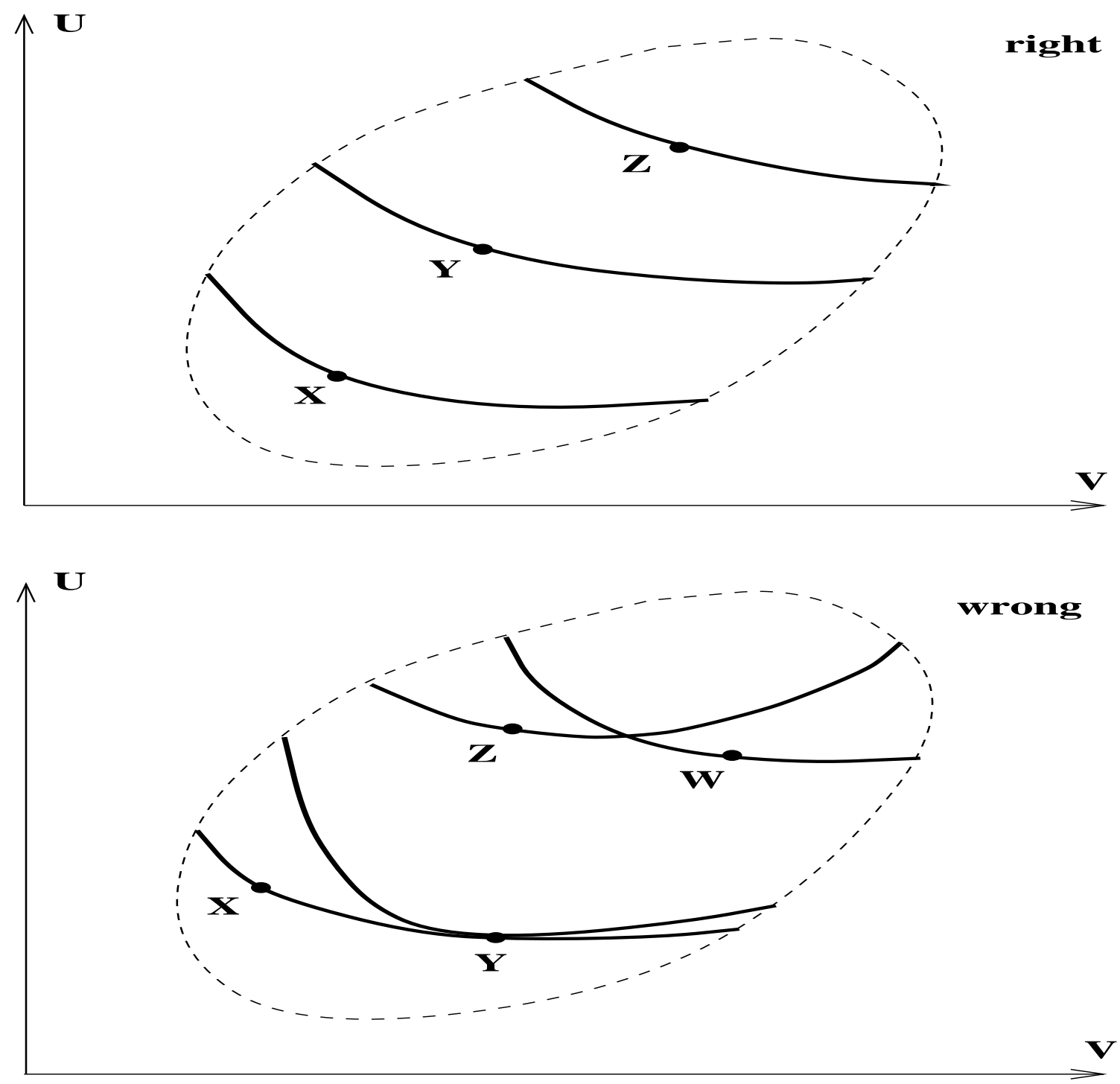

4. The forward sectors of a simple system are nested. The bottom figure shows what could, in principle, could go wrong-but does not. 
It can also be shown from our axioms that the orientation of forward sectors w.r.t. the energy axis is the same for all simple systems. By convention we choose the direction of the energy axis so that the the energy always increases in adiabatic processes at fixed work coordinates. When temperature is defined later, this will imply that temperature is always positive.

Theorem 3 implies that $Y$ is on the boundary of $A_{X}$ if and only if $X$ is on the boundary of $A_{Y}$. Thus the adiabats, i.e., the $\stackrel{\text { A }}{\sim}$ equivalence classes, consist of these boundaries.

Before leaving the subject of simple systems let us remark on the connection with Carathéodory's development. The point of contact is the fact that $X \in \partial A_{X}$. We assume that $A_{X}$ is convex and use transitivity and Lipschitz continuity to arrive, eventually, at Theorem 3. Carathéodory uses Frobenius's theorem, plus assumptions about differentiability to conclude the existence - locally - of a surface containing $X$. Important global information, such as Theorem 3, are then not easy to obtain without further assumptions, as discussed, e.g., in [1].

The next topic is thermal contact and the zeroth law, which entails the very special assumptions about $\prec$ that we mentioned earlier. It will enable us to establish $\mathrm{CH}$ for products of several systems, and thereby show, via Theorem 2, that entropy exists and is additive. Although we have established $\mathrm{CH}$ for a simple system, $\Gamma$, we have not yet established $\mathrm{CH}$ even for a product of two copies of $\Gamma$. This is needed in the definition of $S$ given in (9). The $S$ in (9) is determined up to an affine shift and we want to be able to calibrate the entropies (i.e., adjust the multiplicative and additive constants) of all systems so that they work together to form a global $S$ satisfying the entropy principle. We need five more axioms. They might look a bit abstract, so a few words of introduction might be helpful.

In order to relate systems to each other, in the hope of establishing $\mathrm{CH}$ for compounds, and thereby an additive entropy function, some way must be found to put them into contact with each other. Heuristically, we imagine two simple systems (the same or different) side by side, and fix the work coordinates (e.g., the volume) of each. Connect them with a "copper thread" and wait for equilibrium to be established. The total energy $U$ will not change but the individual energies, $U_{1}$ and $U_{2}$ will adjust to values that depend on $U$ and the work coordinates. This new system (with the thread permanently connected) then behaves like a simple system (with one energy coordinate) but with several work coordinates (the union of the two work coordinates). Thus, if we start initially with $X_{1}=\left(U_{1}, V_{1}\right)$ for system 1 and $X_{2}=\left(U_{2}, V_{2}\right)$ for system 2 , and if we end up with $X=\left(U, V_{1}, V_{2}\right)$ for the new system, we can say that $\left(X_{1}, X_{2}\right) \prec X$. This holds for every choice of $U_{1}$ and $U_{2}$ whose sum is $U$. Moreover, after thermal equilibrium is reached, the two systems can be disconnected, if we wish, and once more form a compound system, whose component parts we say are in thermal equilibrium. That this is transitive is the 
zeroth law.

Thus, we can not only make compound systems consisting of independent subsystems (which can interact, but separate again), we can also make a new simple system out of two simple systems. To do this an energy coordinate has to disappear, and thermal contact does this for us. All of this is formalized in the following three axioms.

A11. Thermal contact. For any two simple systems with state-spaces $\Gamma_{1}$ and $\Gamma_{2}$, there is another simple system, called the thermal join of $\Gamma_{1}$ and $\Gamma_{2}$, with state-space

$$
\Delta_{12}=\left\{\left(U, V_{1}, V_{2}\right): U=U_{1}+U_{2} \text { with }\left(U_{1}, V_{1}\right) \in \Gamma_{1},\left(U_{2}, V_{2}\right) \in \Gamma_{2}\right\}
$$

Moreover,

$$
\Gamma_{1} \times \Gamma_{2} \ni\left(\left(U_{1}, V_{1}\right),\left(U_{2}, V_{2}\right)\right) \prec\left(U_{1}+U_{2}, V_{1}, V_{2}\right) \in \Delta_{12} .
$$

A12. Thermal splitting. For any point $\left(U, V_{1}, V_{2}\right) \in \Delta_{12}$ there is at least one pair of states, $\left.\left(U_{1}, V_{1}\right) \in \Gamma_{1},\left(U_{2}, V_{2}\right)\right) \in \Gamma_{2}$, with $U=U_{1}+U_{2}$, such that

$$
\left(U, V_{1}, V_{2}\right) \stackrel{\mathrm{A}}{\sim}\left(\left(U_{1}, V_{1}\right),\left(U_{2}, V_{2}\right)\right)
$$

If $\left(U, V_{1}, V_{2}\right) \stackrel{\text { A }}{\sim}\left(\left(U_{1}, V_{1}\right),\left(U_{2}, V_{2}\right)\right)$ we say that the states $X=\left(U_{1}, V_{1}\right)$ and $Y=$ $\left.\left(U_{2}, V_{2}\right)\right)$ are in thermal equilibrium and write

$$
X \stackrel{\mathrm{T}}{\sim} Y
$$

A13. Zeroth law of thermodynamics. If $X \stackrel{\mathrm{T}}{\sim} Y$ and if $Y \stackrel{\mathrm{T}}{\sim} Z$ then $X \stackrel{\mathrm{I}}{\sim} Z$.

A11 and A12 together say that for each choice of the individual work coordinates there is a way to divide up the energy $U$ between the two systems in a stable manner. A12 is the stability statement, for it says that joining is reversible, i.e., once the equilibrium has been established, one can cut the copper thread and retrieve the two systems back again, but with a special partition of the energies.

This reversibility allows us to think of the thermal join, which is a simple system in its own right, as a special subset of the product system, $\Gamma_{1} \times \Gamma_{2}$, which we call the thermal diagonal. In particular, A12 allows us to prove easily that $X \stackrel{\mathrm{T}}{\sim} \lambda X$ for all $X$ and all $\lambda>0$.

A13 is the famous zeroth law, which says that the thermal equilibrium is transitive, and hence an equivalence relation. Often this law is taken to mean that there the equivalence classes can be labeled by an 'empirical' temperature, but we do not want to mention temperature at all at this point. It will appear later.

Two more axioms are needed. 
A14 requires that for every adiabat (i.e., an equivalence class w.r.t. $\stackrel{\text { A }}{\sim}$ ) there exists at least one isotherm (i.e., an equivalence class w.r.t. $\stackrel{\mathbb{T}}{\sim}$ ), containing points on both sides of the adiabat. Note that, for each given $X$, only two points in the entire state space $\Gamma$ are required to have the stated property. This assumption essentially prevents a state-space from breaking up into two pieces that do not communicate with each other. Without it, counterexamples to $\mathrm{CH}$ for compound systems can be constructed. A14 implies A8, but we listed A8 separately in order not to confuse the discussion of simple systems with thermal equilibrium.

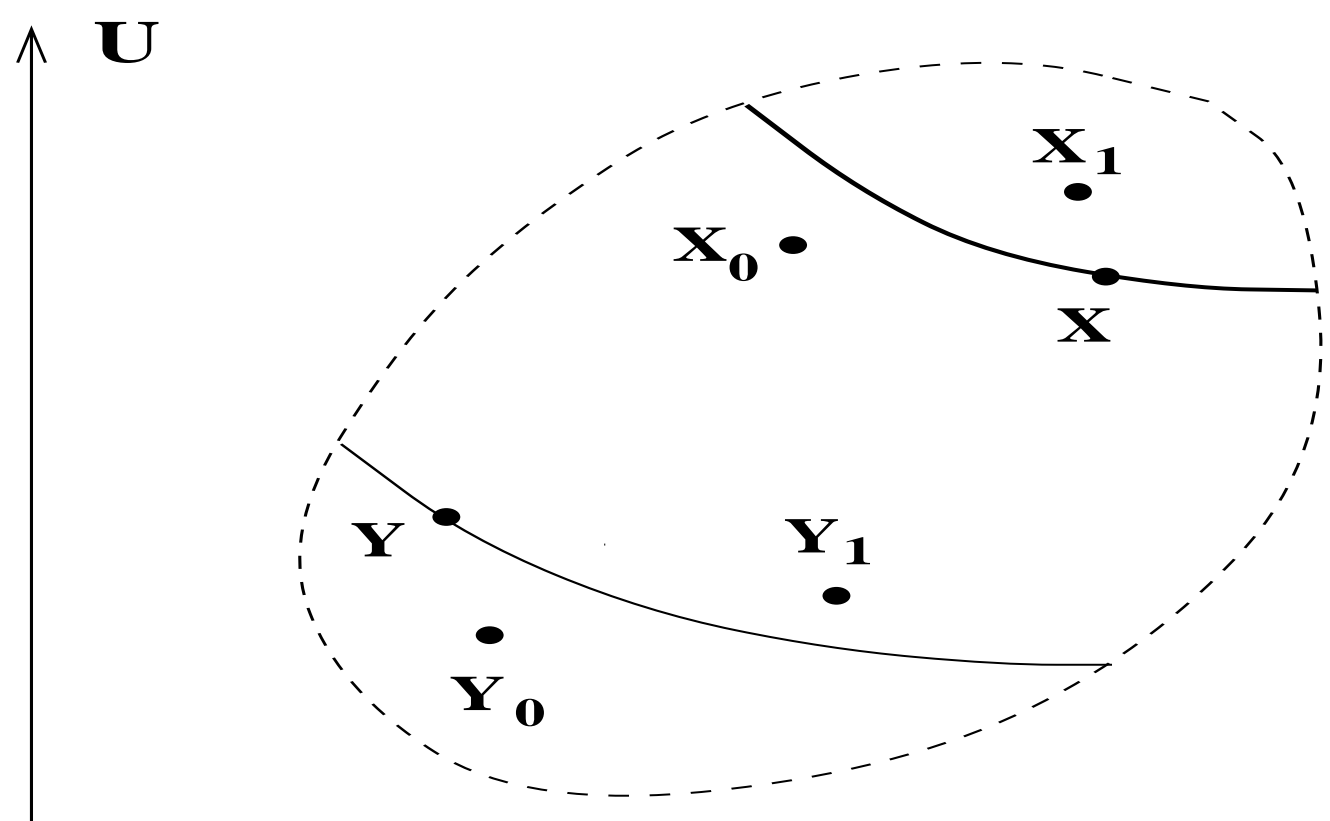

Figure 5. Transversality, A14, requires that each $X$ have points on each side of its adiabat that are in thermal equilibrium.

A15 is a technical and perhaps can be eliminated. Its physical motivation is that a sufficiently large copy of a system can act as a heat bath for other systems. When temperature is introduced later, A15 will have the meaning that all systems have the same temperature range. This postulate is needed if we want to be able to bring every system into thermal equilibrium with every other system.

A14. Transversality. If $\Gamma$ is the state space of a simple system and if $X \in \Gamma$, then there exist states $X_{0} \stackrel{\mathrm{T}}{\sim} X_{1}$ with $X_{0} \prec \prec X \prec \prec X_{1}$.

A15. Universal temperature range. If $\Gamma_{1}$ and $\Gamma_{2}$ are state spaces of simple systems then, for every $X \in \Gamma_{1}$ and every $V$ belonging to the projection of $\Gamma_{2}$ onto the space of its work coordinates, there is a $Y \in \Gamma_{2}$ with work coordinates $V$ such that $X \stackrel{\mathrm{T}}{\sim} Y$.

The reader should note that the concept 'thermal contact' has appeared, but not tem- 
perature or hot and cold or anything resembling the Clausius or Kelvin-Planck formulations of the second law. Nevertheless, we come to the main achievement of our approach: With these axioms we can establish $\mathrm{CH}$ for products of simple systems (each of which satisfies $\mathrm{CH}$, as we already know). First, the thermal join establishes $\mathrm{CH}$ for the (scaled) product of a simple system with itself. The basic idea here is that the points in the product that lie on the thermal diagonal are comparable, since points in a simple system are comparable. In particular, with $X, X_{0}, X_{1}$ as in A14, the states $\left((1-\lambda) X_{0}, \lambda X_{1}\right)$ and $((1-\lambda) X, \lambda X)$ can be regarded as states of the same simple system and are, therefore, comparable. This is the key point needed for the construction of $S$, according to (9). The importance of transversality is thus brought into focus.

With some more work we can establish $\mathrm{CH}$ for multiple scaled copies of a simple system. Thus, we have established $S$ within the context of one system and copies of the system, i.e. condition (ii) of Theorem 1. As long as we stay within such a group of systems there is no way to determine the unknown multiplicative or additive entropy constants. The next task is to show that the multiplicative constants can be adjusted to give a universal entropy valid for copies of different systems, i.e. to establish the hypothesis of Theorem 2. This is based on the following.

LEMMA 4 (Existence of calibrators). If $\Gamma_{1}$ and $\Gamma_{2}$ are simple systems, then there exist states $X_{0}, X_{1} \in \Gamma_{1}$ and $Y_{0}, Y_{1} \in \Gamma_{2}$ such that

$$
X_{0} \prec \prec X_{1} \quad \text { and } \quad Y_{0} \prec \prec Y_{1}
$$

and

$$
\left(X_{0}, Y_{1}\right) \stackrel{\mathrm{A}}{\sim}\left(X_{1}, Y_{0}\right)
$$

The significance of Lemma 4 is that it allows us to fix the multiplicative constants by the condition

$$
S_{1}\left(X_{0}\right)+S_{2}\left(Y_{1}\right)=S_{1}\left(X_{1}\right)+S_{2}\left(Y_{0}\right)
$$

The proof of Lemma 4 is complicated and really uses all the axioms A1 to A14. With its aid we arrive at our chief goal, which is $\mathrm{CH}$ for compound systems.

THEOREM 4 (Entropy principle in products of simple systems). The comparison hypothesis $\mathrm{CH}$ is valid in arbitrary scaled products of simple systems. Hence, by Theorem 2, the relation $\prec$ among states in such state-spaces is characterized by an entropy function $S$. The entropy function is unique, up to an overall multiplicative constant and one additive constant for each simple system under consideration.

At last, we are now ready to define temperature. Concavity of $S$ (implied by A7), Lipschitz continuity of the pressure and the transversality condition, together with some 
real analysis, play key roles in the following, which answers questions Q3 and Q4 posed at the beginning.

THEOREM 5 (Entropy defines temperature.) The entropy, $S$, is a concave and continuously differentiable function on the state space of a simple system. If the function $T$ is defined by

$$
\frac{1}{T}:=\left(\frac{\partial S}{\partial U}\right)_{V}
$$

then $T>0$ and $T$ characterizes the relation $\stackrel{\mathcal{T}}{\sim}$ in the sense that $X \stackrel{\mathrm{T}}{\sim} Y$ if and only if $T(X)=T(Y)$. Moreover, if two systems are brought into thermal contact with fixed work coordinates then, since the total entropy cannot decrease, the energy flows from the system with the higher $T$ to the system with the lower $T$.

The temperature need not be a strictly monotone function of $U$; indeed, it is not so in a 'multiphase region'. It follows that $T$ is not always capable of specifying a state, and this fact can cause some pain in traditional discussions of the second law - if it is recognized, which usually it is not.

Mixing and Chemical Reactions. The core results of our analysis have now been presented and readers satisfied with the entropy principle in the form of Theorem 4 may wish to stop at this point. Nevertheless, a nagging doubt will occur to some, because there are important adiabatic processes in which systems are not conserved, and these processes are not yet covered in the theory. A critical study of the usual textbook treatments should convince the reader that this subject is not easy, but in view of the manifold applications of thermodynamics to chemistry and biology it is important to tell the whole story and not ignore such processes.

One can formulate the problem as the determination of the additive constants $B(\Gamma)$ of Theorem 2. As long as we consider only adiabatic processes that preserve the amount of each simple system (i.e., such that Eqs. (6) and (8) hold), these constants are indeterminate. This is no longer the case, however, if we consider mixing processes and chemical reactions (which are not really different, as far as thermodynamics is concerned.) It then becomes a nontrivial question whether the additive constants can be chosen in such a way that the entropy principle holds. Oddly, this determination turns out to be far more complex, mathematically and physically than the determination of the multiplicative constants (Theorem 2). In traditional treatments one usually resorts to gedanken experiments involving strange, nonexistent objects called 'semipermeable' membranes and 'van t'Hofft boxes'. We present here a general and rigorous approach which avoids all this.

What we already know is that every system has a well-defined entropy function, e.g., for each $\Gamma$ there is $S_{\Gamma}$, and we know from Theorem 2 that the multiplicative constants $a_{\Gamma}$ can been determined in such a way that the sum of the entropies increases in any adiabatic 
process in any compound space $\Gamma_{1} \times \Gamma_{2} \times \ldots$ Thus, if $X_{i} \in \Gamma_{i}$ and $Y_{i} \in \Gamma_{i}$ then

$$
\left(X_{1}, X_{2}, \ldots\right) \prec\left(Y_{1}, Y_{2}, \ldots\right) \quad \text { if and only if } \quad \sum_{i} S_{i}\left(X_{i}\right) \leq \sum_{j} S_{j}\left(Y_{j}\right)
$$

where we have denoted $S_{\Gamma_{i}}$ by $S_{i}$ for short. The additive entropy constants do not matter here since each function $S_{i}$ appears on both sides of this inequality. It is important to note that this applies even to processes that, in intermediate steps, take one system into another, provided the total compound system is the same at the beginning and at the end of the process.

The task is to find constants $B(\Gamma)$, one for each state space $\Gamma$, in such a way that the entropy defined by

$$
S(X):=S_{\Gamma}(X)+B(\Gamma) \quad \text { for } \quad X \in \Gamma
$$

satisfies

$$
S(X) \leq S(Y)
$$

whenever

$$
X \prec Y \quad \text { with } \quad X \in \Gamma, Y \in \Gamma^{\prime} .
$$

Additionally, we require that the newly defined entropy satisfies scaling and additivity under composition. Since the initial entropies $S_{\Gamma}(X)$ already satisfy them, these requirements become conditions on the additive constants $B(\Gamma)$ :

$$
B\left(\Gamma_{1}^{\left(\lambda_{1}\right)} \times \Gamma_{2}^{\left(\lambda_{2}\right)}\right)=\lambda_{1} B\left(\Gamma_{1}\right)+\lambda_{2} B\left(\Gamma_{2}\right)
$$

for all state spaces $\Gamma_{1}, \Gamma_{2}$ under considerations and $\lambda_{1}, \lambda_{2}>0$. Some reflection shows us that consistency in the definition of the entropy constants $B(\Gamma)$ requires us to consider all possible chains of adiabatic processes leading from one space to another via intermediate steps. Moreover, the additivity requirement leads us to allow the use of a 'catalyst' in these processes, i.e., an auxiliary system, that is recovered at the end, although a state change within this system might take place. With this in mind we define quantities $F\left(\Gamma, \Gamma^{\prime}\right)$ that incorporate the entropy differences in all such chains leading from $\Gamma$ to $\Gamma^{\prime}$. These are built up from simpler quantities $D\left(\Gamma, \Gamma^{\prime}\right)$, which measure the entropy differences in onestep processes, and $E\left(\Gamma, \Gamma^{\prime}\right)$, where the 'catalyst' is absent. The precise definitions are as follows. First,

$$
D\left(\Gamma, \Gamma^{\prime}\right):=\inf \left\{S_{\Gamma^{\prime}}(Y)-S_{\Gamma}(X): X \in \Gamma, Y \in \Gamma^{\prime}, X \prec Y\right\}
$$

If there is no adiabatic process leading from $\Gamma$ to $\Gamma^{\prime}$ we put $D\left(\Gamma, \Gamma^{\prime}\right)=\infty$. Next, for any given $\Gamma$ and $\Gamma^{\prime}$ we consider all finite chains of state spaces, $\Gamma=\Gamma_{1}, \Gamma_{2}, \ldots, \Gamma_{N}=\Gamma^{\prime}$ such that $D\left(\Gamma_{i}, \Gamma_{i+1}\right)<\infty$ for all $\mathrm{i}$, and we define

$$
E\left(\Gamma, \Gamma^{\prime}\right):=\inf \left\{D\left(\Gamma_{1}, \Gamma_{2}\right)+\cdots+D\left(\Gamma_{N-1}, \Gamma_{N}\right)\right\}
$$


where the infimum is taken over all such chains linking $\Gamma$ with $\Gamma^{\prime}$. Finally we define

$$
F\left(\Gamma, \Gamma^{\prime}\right):=\inf \left\{E\left(\Gamma \times \Gamma_{0}, \Gamma^{\prime} \times \Gamma_{0}\right)\right\}
$$

where the infimum is taken over all state spaces $\Gamma_{0}$. (These are the 'catalysts'.)

The importance of the $F$ 's for the determination of the additive constants is made clear in the following theorem:

THEOREM 6 (Constant entropy differences). If $\Gamma$ and $\Gamma^{\prime}$ are two state spaces then for any two states $X \in \Gamma$ and $Y \in \Gamma^{\prime}$

$$
X \prec Y \quad \text { if and only if } \quad S_{\Gamma}(X)+F\left(\Gamma, \Gamma^{\prime}\right) \leq S_{\Gamma^{\prime}}(Y)
$$

An essential ingredient for the proof of this theorem is Eq. (20).

According to Theorem 6 the determination of the entropy constants $B(\Gamma)$ amounts to satisfying the inequalities

$$
-F\left(\Gamma^{\prime}, \Gamma\right) \leq B(\Gamma)-B\left(\Gamma^{\prime}\right) \leq F\left(\Gamma, \Gamma^{\prime}\right)
$$

together with the linearity condition (23). It is clear that (28) can only be satisfied with finite constants $B(\Gamma)$ and $B\left(\Gamma^{\prime}\right)$, if $F\left(\Gamma, \Gamma^{\prime}\right)>-\infty$. To exclude the pathological case $F\left(\Gamma, \Gamma^{\prime}\right)=-\infty$ we introduce our last axiom A16, whose statement requires the following definition.

Definition. A state-space, $\Gamma$ is said to be connected to another state-space $\Gamma^{\prime}$ if there are states $X \in \Gamma$ and $Y \in \Gamma^{\prime}$, and state spaces $\Gamma_{1}, \ldots, \Gamma_{N}$ with states $X_{i}, Y_{i} \in \Gamma_{i}$, $i=1, \ldots, N$, and a state space $\Gamma_{0}$ with states $X_{0}, Y_{0} \in \Gamma_{0}$, such that

$$
\left(X, X_{0}\right) \prec Y_{1}, \quad X_{i} \prec Y_{i+1}, i=1, \ldots, N-1, \quad X_{N} \prec\left(Y, Y_{0}\right)
$$

A16. (Absence of sinks): If $\Gamma$ is connected to $\Gamma^{\prime}$ then $\Gamma^{\prime}$ is connected to $\Gamma$.

This axiom excludes $F\left(\Gamma, \Gamma^{\prime}\right)=-\infty$ because, on general grounds, one always has

$$
-F\left(\Gamma^{\prime}, \Gamma\right) \leq F\left(\Gamma, \Gamma^{\prime}\right)
$$

Hence $F\left(\Gamma, \Gamma^{\prime}\right)=-\infty$ (which means, in particular, that $\Gamma$ is connected to $\Gamma^{\prime}$ ) would imply $F\left(\Gamma^{\prime}, \Gamma\right)=\infty$, i.e., that there is no way back from $\Gamma^{\prime}$ to $\Gamma$. This is excluded by Axiom 16 .

The quantities $F\left(\Gamma, \Gamma^{\prime}\right)$ have simple subadditivity properties that allow us to use the Hahn-Banach theorem to satisfy the inequalities $(28)$, with constants $B(\Gamma)$ that depend linearly on $\Gamma$, in the sense of Eq. (23). Hence we arrive at 
THEOREM 7 (Universal entropy). The additive entropy constants of all systems can be calibrated in such a way that the entropy is additive and extensive, and $X \prec Y$ implies $S(X) \leq S(Y)$, even when $X$ and $Y$ do not belong to the same state space.

Our final remark concerns the remaining non-uniqueness of the constants $B(\Gamma)$. This indeterminacy can be traced back to the non-uniqueness of a linear functional lying between $-F\left(\Gamma^{\prime}, \Gamma\right)$ and $F\left(\Gamma, \Gamma^{\prime}\right)$ and has two possible sources: One is that some pairs of state-spaces $\Gamma$ and $\Gamma^{\prime}$ may not be connected, i.e., $F\left(\Gamma, \Gamma^{\prime}\right)$ may be infinite (in which case $F\left(\Gamma^{\prime}, \Gamma\right)$ is also infinite by axiom A16). The other is that there might be a true gap, i.e.,

$$
-F\left(\Gamma^{\prime}, \Gamma\right)<F\left(\Gamma, \Gamma^{\prime}\right)
$$

might hold for some state spaces, even if both sides are finite.

In nature only states containing the same amount of the chemical elements can be transformed into each other. Hence $F\left(\Gamma, \Gamma^{\prime}\right)=+\infty$ for many pairs of state spaces, in particular, for those that contain different amounts of some chemical element. The constants $B(\Gamma)$ are, therefore, never unique: For each equivalence class of state spaces (with respect to the relation of connectedness) one can define a constant that is arbitrary except for the proviso that the constants should be additive and extensive under composition and scaling of systems. In our world there are 92 chemical elements (or, strictly speaking, a somewhat larger number, $N$, since one should count different isotopes as different elements), and this leaves us with at least 92 free constants that specify the entropy of one gram of each of the chemical elements in some specific state.

The other possible source of non-uniqueness, a nontrivial gap (32) for systems with the same composition in terms of the chemical elements is, as far as we know, not realized in nature. (Note that this assertion can be tested experimentally without invoking semipermeable membranes.) Hence, once the entropy constants for the chemical elements have been fixed and a temperature unit has been chosen (to fix the multiplicative constants) the universal entropy is completely fixed.

We are indebted to many people for helpful discussions, including Fred Almgren, Thor Bak, Bernard Baumgartner, Pierluigi Contucci, Roy Jackson, Anthony Knapp, Martin Kruskal, Mary Beth Ruskai and Jan Philip Solovej.

\section{REFERENCES}

[1] Boyling, J.B., 1972 An axiomatic approach to classical thermodynamics, Proc. Roy. Soc. London A329, 35-70.

[2] Buchdahl, H. A., 1966, The Concepts of Classical Thermodynamics, (Cambridge University Press, Cambridge). 
[3] Carathéodory, C., 1909 Untersuchung über die Grundlagen der Thermodynamik, Math. Annalen 67, 355-386.

[4] Cooper, J.L.B., 1967 The foundations of thermodynamics, Jour. Math. Anal. and Appl. 17, 172-193.

[5] Duistermaat, J. J., 1968, Energy and entropy as real morphisms for addition and order, Synthese 18, 327-393.

[6] Giles, R., 1964, Mathematical Foundations of Thermodynamics, (Pergamon, Oxford).

[7] Lieb, E.H. and Yngvason, J., The Physics and Mathematics of the Second Law of Thermodynamics, Preprint (1997). Physics Reports (in press). Austin Math. Phys. archive 97-457. Los Alamos archive cond-mat/9708200.

[8] Planck, M., 1926 Über die Begrundung des zweiten Hauptsatzes der Thermodynamik, Sitzungsber. Preuss. Akad. Wiss., Phys. Math. Kl., 453-463.

[9] Roberts, F. S. and Luce, R. D., 1968, Axiomatic thermodynamics and extensive measurement, Synthese 18, 311-326. 